\title{
Novel classical ground state of a many body system in arbitrary dimensions
}

\author{
G. Date*, Pijush K. Ghosh ${ }^{\dagger}$, and M. V. N. Murthy \\ The Institute of Mathematical Sciences, Madras 600 113, India.
}

(July 31, 2021)

The classical ground state of a D- dimensional many body system with two and three body interactions is studied as a function of the strength of the three body interaction. We prove exactly that beyond a critical strength of the three body interaction, the classical ground state of the system is one in which all the particles are on a line. The positions of the particles in this string configuration are uniquely determined by the zeros of the Hermite polynomials.

PACS numbers: 03,20.+i, 03.65.Ge, 24.10.Cn

In this letter we consider the following Hamiltonian which displays remarkable classical properties. The classical Hamiltonian of the model for $N$ interacting particles, in $D$ dimensions, is,

$$
\begin{aligned}
H & =\frac{1}{2} \sum_{i=1}^{N}\left[p_{i}^{2}+r_{i}^{2}\right]+\frac{g_{1}}{2} \sum_{i, j(j \neq i)}^{N} \frac{1}{r_{i j}^{2}} \\
& +\frac{g_{2}}{2} \sum_{i, j, k(i \neq j \neq k)}^{N} \frac{\vec{r}_{i j} \cdot \vec{r}_{i k}}{r_{i j}^{2} r_{i k}^{2}},
\end{aligned}
$$

where $g_{1}$ and $g_{2}$ are in general arbitrary but positive coupling strengths of the two and three-body interactions. We have set the mass $m=1$, oscillator frequency $\omega=1$ and $\vec{r}_{i j}=\vec{r}_{i}-\vec{r}_{j}$.

We prove exactly that when $g_{2}>g_{1}$ the classical ground state of this Hamiltonian is unique (modulo rotations) and is described by all the particles along a line with locations determined by the zeros of the Hermite polynomial $H_{N}$.

There are several reasons to consider this Hamiltonian. The model Hamiltonian given above has a very interesting limit. When

$$
g_{1}=g(g+D-2), \quad g_{2}=g^{2},
$$

it has been shown that the quantum mechanical ground state and an infinite tower of excited states may be obtained exactly in D-dimensions [1,2]. ( In D=2, some of these solutions may be obtained from the Hamiltonian of the anyons in two dimensions in the zero total angular momentum sector [3].) In particular for the ground state solving the Schroedinger equation $H \psi_{0}=E_{0} \psi_{0}$ we obtain,

$$
\psi_{0}=\prod_{i<j}\left|\vec{r}_{i j}\right|^{g} \exp \left(-\frac{1}{2} \sum_{i} r_{i}^{2}\right)
$$

and the quantum ground state energy is given by,

$$
E_{0}=\frac{1}{2}[N D+g N(N-1)],
$$

where $g \geq 0$. In the limit $g \rightarrow 0$, the system describes $N$ free bosons confined in a harmonic oscillator potential without the centrifugal barrier.

For $D=1$, the model is the celebrated CalogeroSutherland model (CSM) [4]. The three-body term is identically zero in this case. It is known that the classical ground state of CSM is the one in which the location of the $N$ particles are determined by the zeros of the Hermite polynomial $H_{N}$ [5]. The CSM is of course exactly solvable quantum mechanically.

For $D=2$, when $g_{2}=0$, the model reduces to a model with only two-body inverse square interaction which has some interesting applications to quantum dots and is considered in detail in ref. [6]. Unlike in one dimension, however, the three body term does not vanish in higher dimensions and plays a crucial role in the analysis that is to follow. Similar to the one dimensional CSM the norm of the ground state wave function in eq.(3), when $g^{2}=g_{1}=g_{2}$, can be identified with the joint probability density function of the eigenvalues of complex random matrices for a particular value of $g$ [1, 凡]. Also, the ground state wave function is exactly found to be the gauge transformed Laughlin's wave function and exhibits off-diagonal long range order [8]. For arbitrary $g_{1}, g_{2}$, however, the quantum problem may not be solvable even for the ground state.

We also note that the three-body interactions have been used in several areas like nuclear, atomic and condensed matter physics. The main reason usually is to account for the many-body effects in systems of particles or clusters. It is also known that the three-body interactions may be used to bring about structural transitions in the classical ground state. Recently, Weber and Stillinger [9] have proposed a form of the three-body interaction for inducing structural transitions in the classical ground state of many particle systems in their molecular dynamics simulation. The three-body interaction used here bears a resemblance to the one proposed by Weber and Stillinger.

In what follows we keep $g_{1}$ and $g_{2}$ arbitrary but positive and analyse the classical ground state of the Hamiltonian in eq.(1) in $D$-dimensions. Defining

$$
g_{1}=g^{2}, \quad g_{2}=g^{2}+\lambda,
$$

the Hamiltonian can be written in the form, 


$$
H=\frac{1}{2} \sum_{i=1}^{N}\left[p_{i}{ }^{2}+R_{i}^{2}\right]+g \frac{N(N-1)}{2}+\frac{\lambda}{2} V_{3},
$$

where

$$
V_{3}=\sum_{i, j, k(i \neq j \neq k)}^{N} \frac{\vec{r}_{i j} \cdot \vec{r}_{i k}}{r_{i j}^{2} r_{i k}^{2}}
$$

and

$$
\vec{R}_{i}=\vec{r}_{i}-g \sum_{j(j \neq i)} \frac{\vec{r}_{i j}}{r_{i j}^{2}} .
$$

Therefore $\lambda=0$ corresponds to the special case when $g_{1}=g_{2}$. Here $g$ may be chosen to be either positive or negative. The results that follow, however, cannot dependent on this choice since the Hamiltonian and the equilibrium conditions depend only on $g^{2}$. We choose to work with $g>0$ since the analysis is simpler. The classical equilibrium configurations(fixed points) are obtained by solving,

$$
\begin{aligned}
\vec{p}_{k} & =0, \\
\sum_{j} \vec{R}_{j} \cdot\left(\nabla_{k} \vec{R}_{j}\right)+\frac{\lambda}{2} \nabla_{k} V_{3} & =0,
\end{aligned}
$$

where $k=1, \ldots, N$ and the derivatives are taken with respect to configuration coordinates $\vec{r}_{k}$. The energy at any of these extrema are given by,

$$
E=\sum_{i=1}^{N} \frac{1}{2} R_{i}^{2}+g \frac{N(N-1)}{2}+\frac{\lambda}{2} V_{3}=\sum_{i=1}^{N} r_{i}^{2},
$$

where $\vec{r}_{i}$ are solutions of the eq. (10). The last part of the above equation follows from the fact that the solutions of the equilibrium equations imply, in general,

$$
\sum_{i=1}^{N} \frac{1}{2} r_{i}^{2}=\frac{g^{2}}{2} \sum_{i, j(j \neq i)} \frac{1}{r_{i j}^{2}}+\frac{1}{2}\left(g^{2}+\lambda\right) \sum_{i \neq j \neq k} \frac{\vec{r}_{i j} \cdot \vec{r}_{i k}}{r_{i j}^{2} r_{i k}^{2}} .
$$

Thus the interaction energy is equal to the confinement energy for all equilibrium configurations.

Before analysing the Hamiltonian further we first establish properties of the three-body potential $V_{3}$. Symmetrising $V_{3}$ explicitly, we have

$$
V_{3}=\frac{1}{3} \sum_{i, j, k(i \neq j \neq k)}^{N}\left[\frac{\vec{r}_{i j} \cdot \vec{r}_{i k}}{r_{i j}^{2} r_{i k}^{2}}+\frac{\vec{r}_{j k} \cdot \vec{r}_{j i}}{r_{j k}^{2} r_{j i}^{2}}+\frac{\vec{r}_{k i} \cdot \vec{r}_{k j}}{r_{k i}^{2} r_{k j}^{2}}\right],
$$

and using the fact that $\vec{r}_{i j}+\vec{r}_{j k}+\vec{r}_{k i}=0$, it is easy to show that

$$
\begin{aligned}
V_{3} & =\frac{2}{3} \sum_{i, j, k(i \neq j \neq k)}^{N} \frac{r_{i j}^{2} r_{j k}^{2}-\left(\vec{r}_{i j} \cdot \vec{r}_{j k}\right)^{2}}{r_{i j}^{2} r_{j k}^{2} r_{k i}^{2}} \\
& =\frac{2}{3} \sum_{i, j, k(i \neq j \neq k)}^{N} \frac{\sin ^{2} \theta_{i j k}}{\left(\vec{r}_{i j}+\vec{r}_{j k}\right)^{2}},
\end{aligned}
$$

where $\theta_{i j k}$ is the angle between $\vec{r}_{i j}$ and $\vec{r}_{j k}$. From eq.(14) following results follow independent of whether $\vec{r}_{i}$ are solutions of the equilibrium equations or not:

1. Clearly $V_{3} \geq 0$ for all $\vec{r}_{i}$.

2. Further $V_{3}=0$ means that each term in the summation must be zero since each term is separately a square. Therefore, $\theta_{i j k}$ must be either zero or $\pi$. This means that all particles must be on line when $V_{3}$ attains its global minimum.

3. By a similar calculation it is easy to see that, $\nabla_{i} V_{3}=0$ for all $i$ if all the particles are on a line, that is, $\vec{r}_{i}=s_{i} \hat{e}$, where the unit vector $\hat{e}$ denotes an arbitrary direction in the $D$ dimensional configuration space.

4. Therefore, if $\vec{r}_{i}=s_{i} \hat{e}$ is an extremum then the solutions of eq.(10) as well as the energy in eq.(11) are independent of $\lambda$. Furthermore this energy attains its absolute minimum value namely,

$$
E_{\text {line }}=g N(N-1) / 2 \quad \text { iff } \quad \vec{R}_{i}=0 \quad \forall \quad i .
$$

To compare qualitative features for $\lambda$ positive, negative or zero we consider special configurations such that all the particles lie on a plane passing through the origin. The plane in fact can be chosen to be the x-y plane (say) because of the overall rotational invariance. The equilibrium configurations among these are governed by the same equations. Of these planer configurations we consider two special ones which are also solutions of the equilibrium equations, namely, (1) all $\mathrm{N}$ particles on a circle with centre at the origin and (2) (N-1) particles on a circle and one at the centre. For these configurations the respective energies are given by,

$$
\begin{gathered}
E_{\bigcirc}=E_{\text {line }} \sqrt{1+\frac{2 \lambda}{3 g^{2}} \frac{N-2}{N-1}}, \\
E_{\odot}=E_{\text {line }} \sqrt{1+\frac{2 \lambda}{3 g^{2}} \frac{(N+4)(N-3)}{N^{2}}},
\end{gathered}
$$

where $E_{\text {line }}$ is given in eq.(15) and refers to the energy of the line configuration(which is independent of $\lambda$ ).

(a) $\lambda<0$ : For this case the line configuration can not attain the absolute minimum of the energy since the special configurations mentioned above have lower energy. For arbitrary $N$ the ground state is indeed hard to find though it is clear from the form of $V_{3}$ that these are necessarily non one dimensional configurations. Among the sub-class of planer configurations, or equivalently $D=2$, we know that for $N \leq 5$ circle configuration has the lowest energy where as for $6 \leq N \leq 8$ circle-dot has the 
lowest energy. For $N \geq 9$ multishell configurations have the lowest energy 10 . For $D>2$, of course these in general are not classical ground states. Note that even though $\lambda<0$, by definition $\lambda>-g^{2}$ since the strength of the three body term is necessarily positive. This ensures that the energy is real in these configurations.

(b) $\lambda=0$ : The absolute minimum of energy is attained for all configurations satisfying the equation $\vec{R}_{i}=0$. The energy in all these configurations is given by,

$$
E=g \frac{N(N-1)}{2}=E_{\text {line }}
$$

In this case the line, the circle and the circle-dot are all degenerate. Within the sub-class of planer configuration (equivalently for $D=2$ ) numerical simulations show that there are many other configurations which are also degenerate. For $D=2$, the quantum mechanical ground state can be obtained exactly in this limit as noted at the beginning. The classical energy is the same as the quantum mechanical ground state energy apart from the contribution from the zero point fluctuation. Note that this is rather special for $D=2$.

(c) $\lambda>0$ : The line configuration is certainly a ground state since the absolute minimum of the energy is attained. Because $V_{3}$ is positive definite or zero, any other extremum must necessarily have higher energy,

$$
E \geq g \frac{N(N-1)}{2}=E_{\text {line }} \quad \forall \quad \lambda \geq 0 .
$$

Thus for all $\lambda>0$, the classical ground states are line configurations with $\vec{r}_{i}=s_{i} \hat{e}$, where $\hat{e}$ defines an arbitrary direction in the $D$ dimensional space. The positions of the particles on the line, $s_{i}$ are determined by the equation $\vec{R}_{i}=0$. That is,

$$
s_{i}=g \sum_{j(j \neq i)}^{N} \frac{1}{s_{i j}} \quad \forall \quad i=1, \ldots, N
$$

and an absolute minimum is attained when each $s_{i}$ satisfies this equation.

We now show that eqs. 20) in fact have a unique solution. We scale out $g$ by defining a new variable, $t_{i}=g^{-1 / 2} s_{i}$. Define

$$
C_{k}=\sum_{i=1}^{N} t_{i}^{k+1}
$$

Using the equilibrium condition,

$$
C_{k}=\frac{1}{2} \sum_{i, j(i \neq j)} \frac{t_{i}^{k}-t_{j}^{k}}{t_{i j}}=\frac{1}{2} \sum_{i, j(i \neq j)} \sum_{r=0}^{k-1} t_{i}^{r} t_{j}^{k-1-r}
$$

Manipulating the summations, we get the recursion relation,

$$
C_{k+1}=\frac{1}{2}\left[\sum_{r=0}^{k} C_{r-1} C_{k-1-r}-(k+1) C_{k-1}\right] \quad \forall \quad k \geq 0 .
$$

$C_{k}$ are clearly function of only $N$ and are independent of any particular solution of eq.(20). Also, by definition $C_{-1}=N$ and $C_{0}=0$ using directly the eq.(20).

Let $t_{i}^{\prime}$ be another solution of the eq.(20). Because the $C_{k}$ are independent of the particular solution, we have,

$$
C_{k}=\sum_{i=1}^{N} t_{i}^{k+1}=\sum_{i=1}^{N}\left(t^{\prime}\right)_{i}^{k+1}
$$

Therefore,

$$
\sum_{i=1}^{N}\left(t_{i}^{k+1}-\left(t^{\prime}\right)_{i}^{k+1}\right)=\sum_{i} \Delta t_{i} a_{k i}=0
$$

where $\Delta t_{i}=t_{i}-\left(t^{\prime}\right)_{i}$ and $a_{k i}=\sum_{r=0}^{k} t_{i}^{r}\left(t^{\prime}\right)_{i}^{k-r}$. Multiplying both sides by arbitrary $b_{k}$ and summing over $\mathrm{k}$ we get,

$$
\sum_{i} \Delta t_{i} \beta_{i}=0
$$

where $\beta_{i}=\sum_{k=0}^{\infty} a_{k i} b_{k}$. Since $b_{k}$ and therefore $\beta_{i}$ are completely arbitrary we have $\Delta t_{i}=0$. Therefore the solution is unique. It is well known [5], in fact, the Nzeros of the Hermite polynomial $H_{N}(t)$, namely $t_{i}$, satisfy the eq. 20) with $t_{i}=g^{-1 / 2} s_{i}$. Hence the uniqueness proof implies that the positions of the particles along a line in the ground state are determined by the zeros of the Hermite polynomial.

As an aside, we also remark that since $C_{k}$ are constructed out of the zeros of the Hermite polynomials, we have also obtained an infinite set of identities for the zeros of the Hermite polynomial.

This completes the proof that for all $\lambda>0$ there is only one unique global minimum (modulo the states obtained by rotations). The corresponding configuration is a line where the positions of the particles are given as the zeros of Hermite polynomial in terms of the scaled variables $t_{i}$.

We have thus demonstrated that a $D$ dimensional Hamiltonian with two and three body interactions has a unique stringlike ground state when the strength of the three body term exceeds a critical value determined in terms of the parameter $\lambda$. Further we note:

1. The result is independent of the strength of the two-body term, the number of particles and the strength of the oscillator potential. Thus even in the full quantum theory it is possible to choose $g$ or $N$ large enough such that the quantum fluctuations about the classical ground state are as small as we wish. The above statements about the classical ground state approximately remain valid, therefore, even in the quantum theory. 
2. The string like ground state one obtains here for $\lambda>0$ is identical to the classical ground state of the one-dimensional CSM Hamiltonian.

3. The model provides an example of a structural transition driven by the mutual interactions without any external influence. In fact this statement is manifestly independent of the space dimension. Thus we have a powerful result that in any spacedimension, the Hamiltonian in eq.(1) gives rise to string like ground state when the strength of the three body term exceeds the strength of the two body term irrespective of the number of particles, the strength of the confinement potential and the strength of the two body term.

4. Finally, we point out that the form of the threebody potential as given in eq.(14), may be easily generalised. Note that the results of the analysis remain unchanged as long as $V_{3}$ and its gradient vanish on a line and $V_{3}$ is positive semi-definite. In particular,

$$
\begin{aligned}
v_{3}\left(\vec{r}_{i j}, \vec{r}_{j k}, \vec{r}_{k i}\right) & =\left(\vec{r}_{i j} \cdot \vec{r}_{j k}\right)\left(\vec{r}_{j k} \cdot \vec{r}_{k i}\right)+\text { cyclic } \\
& =r_{i j}^{2} r_{j k}^{2} \sin ^{2}\left(\theta_{i j k}\right)
\end{aligned}
$$

satisfies these properties. Hence it follows that for any $V_{3}$ of the form,

$$
V_{3}=\sum_{i \neq j \neq k} f\left(r_{i j}^{2}, r_{j k}^{2}, r_{k i}^{2}\right) v_{3}\left(\vec{r}_{i j}, \vec{r}_{j k}, \vec{r}_{k i}\right)
$$

where $f\left(r_{i j}, r_{i k}, r_{j k}\right)$ is a symmetric function of its arguments and is positive, also vanishes for a line together with its gradient. The $V_{3}$ considered in eq.(14) corresponds to

$$
f\left(r_{i j}, r_{j k}, r_{k i}\right)=\left(r_{i j}^{2} r_{j k}^{2} r_{k i}^{2}\right)^{-1}
$$

Thus, for any arbitrary $f$ modulo these properties, the mere presence of a three-body interaction is sufficient to give a string like ground state. The crucial point here is that the geometric structure of the ground state depends on the $\sin ^{2}\left(\theta_{i j k}\right)$ which favours string configurations and not on the precise form of $f$. The three-body interaction here differs from the one proposed by Weber and Stillinger [9], in that $2 \theta_{i j k}$ appears in place of $\theta_{i j k}$. While the final ground state configuration may be different in these two cases, the presence of the three body term is crucial in defining the actual ground state configuration.

\section{ACKNOWLEDGMENTS}

We thank A. Khare for suggestions and a critical reading of the manuscript. We also thank G. Baskaran, R.
K. Bhaduri, P. P. Divakaran, Tabish Qureishi, Madan Rao, R. Shankar and Surajit Sengupta for discussions and comments.

[1] A. Khare and K. Ray, Phys. Lett. A230, 139(1997).

[2] F. Calogero and C. Marchioro, J. Math. Phys. (N.Y.) 14, 182(1973).

[3] G. Date and M. V. N. Murthy, Phys. Rev. A 48, 105 (1993).

[4] F. Calogero, J. Math. Phys.(N.Y.) 10, 2191(1969); B. Sutherland, J. Math. Phys. (N.Y.) 12, 246(1971); Phys. Rev. A4, 2019(1971); M. A. Olshanetsky and A. M. Perelomov, Phys. Rep. 71, 314(1981), ibid 94, 6(1983).

[5] S. Ahmed, M. Bruschi, F. Calogero, M. A. Olshanetsky and A. M. Perelomov, Il. Nuovo Cimento, 49B, 173(1979); In the context of CSM see also F. Calogero, Lett. Nuovo Cimento,20, 251(1977).

[6] N. F. Johnson and L. Quiroga, Phys. Rev. Lett. 74, 4277(1995); see also J. Phys. Condens. Matt. 9, 5889 (1997) for a detailed study of inverse square interaction.

[7] M. V. Feigel'man and M. A. Skvortsov, Nucl. Phys. B 506 (FS), 665 (1997).

[8] S. Girvin and A. McDonald, Phys. Rev. Lett. 58, 1252 (1987).

[9] T. A. Weber and F. H. Stillinger, Phys. Rev. E48, 4351(1993).

[10] V. M. Bedanov and F. M. Peeters, Phys. Rev. B49, 2667(1994); F. Bolten and U. Rossler, Superlatt. and Microstruct. 13, 140(1993); G. Date, M. V. N. Murthy and Radhika Vathsan, J. Phys. Condens. Matter 10, 5875 (1998). 